\title{
Revealing the Band Structure of FAPI Quantum Dot Film and its Interfaces with Electron and Hole Transport Layer using Time Resolved Photoemission
}

Dylan Amelot ${ }^{1}$, Prachi Rastogi ${ }^{1}$, Bertille Martinez ${ }^{1,2}$, Charlie Gréboval ${ }^{1}$, Clément Livache ${ }^{1,2}$, Francesco Andrea Bresciani ${ }^{1}$, Junling Qu ${ }^{1}$, Audrey Chu ${ }^{1}$, Mayank Goyal ${ }^{1,3}$, Sang-Soo Chee ${ }^{1}$, Nicolas Casaretto ${ }^{1}$, Xiang Zhen $\mathrm{Xu}^{2}$, Christophe Méthivier ${ }^{4}$, Hervé Cruguel ${ }^{1}$, Abdelkarim Ouerghi ${ }^{5}$, Angshuman $\mathrm{Nag}^{3}$, Mathieu G. Silly ${ }^{6}$, Nadine Witkowski ${ }^{1}$, Emmanuel Lhuillier $^{1 *}$

${ }^{1}$ Sorbonne Université, CNRS, Institut des NanoSciences de Paris, INSP, F-75005 Paris, France.

${ }^{2}$ Laboratoire de Physique et d'Etude des Matériaux, ESPCI-Paris, PSL Research University, Sorbonne Université, CNRS UMR 8213, 10 rue Vauquelin 75005 Paris, France.

${ }^{3}$ Department of Chemistry, Indian Institute of Science Education and Research (IISER), Pune 411008, India.

4 Sorbonne Université, UMR CNRS 7197, Laboratoire de Réactivité de Surface, 4 place Jussieu, F-75005 Paris, France

${ }^{5}$ Centre de Nanosciences et de Nanotechnologies, CNRS, University of Paris-Sud, Université Paris-Saclay, C2N, Palaiseau 91120, France.

${ }^{6}$ Synchrotron-SOLEIL, Saint-Aubin, BP48, F91192 Gif sur Yvette Cedex, France.

To whom correspondence should be sent: el@insp.upmc.fr

\section{Table of content}

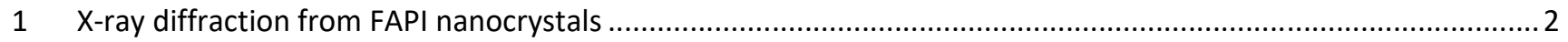

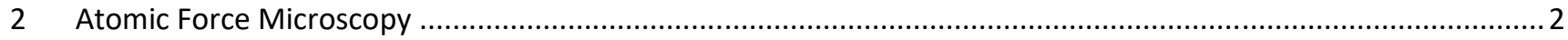

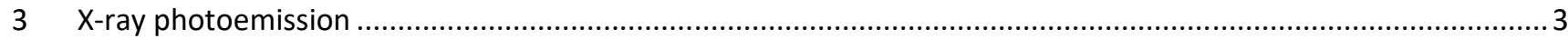

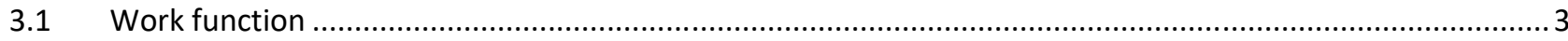

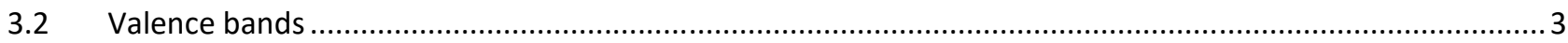

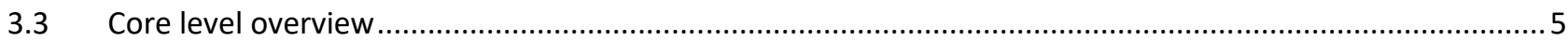

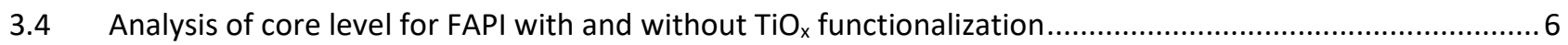

3.5 Analisys of core level for FAPI with and without MoOx functionalization .................................................. 7

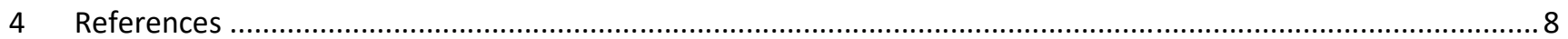




\section{X-ray diffraction from FAPI nanocrystals}

X-ray diffraction of a FAPI nanocrystals film is given in Figure S 1. X-ray diffraction is done on site at INSP using a Rigaku Smartlab diffractometer, in $\theta-2 \theta$ geometry. The diffraction pattern is consistent with the cubic phase of FAPI.



Figure S 1 X-ray diffraction pattern from a FAPI nanocrystal film.

\section{Atomic Force Microscopy}

Atomic Force microscopy (AFM) image have been used to reveal the surface roughness of FAPI nanocrystals film before and after their functionalization by $\mathrm{TiO}_{x}$ and $\mathrm{MoO}_{x}$, see Figure $\mathrm{S} 2$. Images were recorded using Multimode8 from Brüker, in peak force mode.
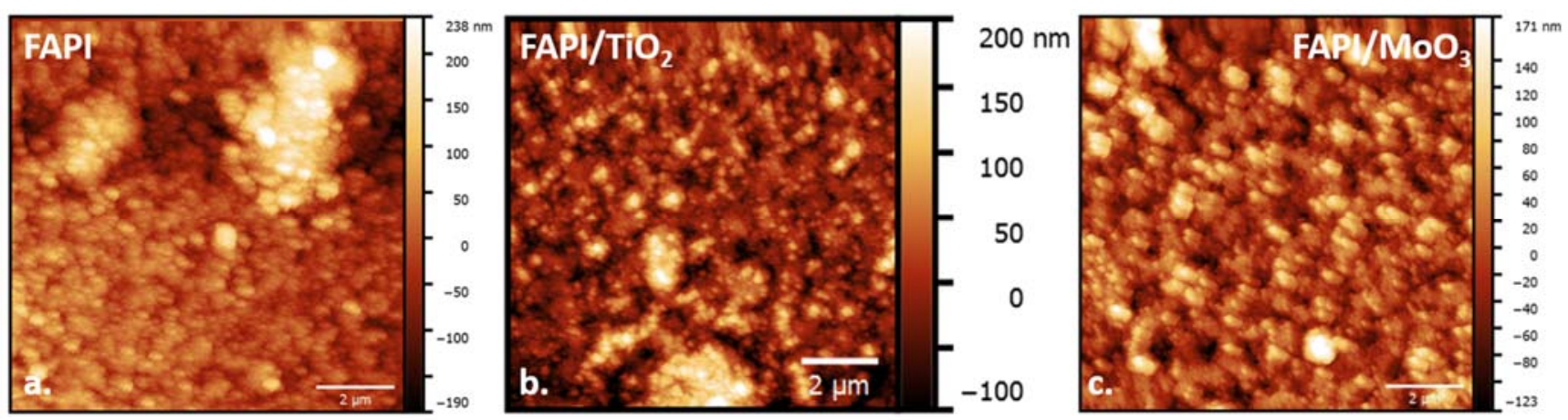

Figure 2 AFM images of the FAPI nanocrystals film (a.), FAPI nanocrystals film with $\mathrm{TiO}_{2}$ deposited on the top (b) and FAPI nanocrystals film with $\mathrm{MoO}_{3}$ deposited on the top (c.). The associated average roughnesses are of $48 \mathrm{~nm}$ (a), $37 \mathrm{~nm}$ (b) and $35 \mathrm{~nm}$ (c). 


\section{X-ray photoemission}

\subsection{Work function}

The work function of FAPI has been determined using the cut-off of the secondary electron. It reveals that vacuum level is $4.05 \mathrm{eV}$ above the Fermi level, see Figure S 3.

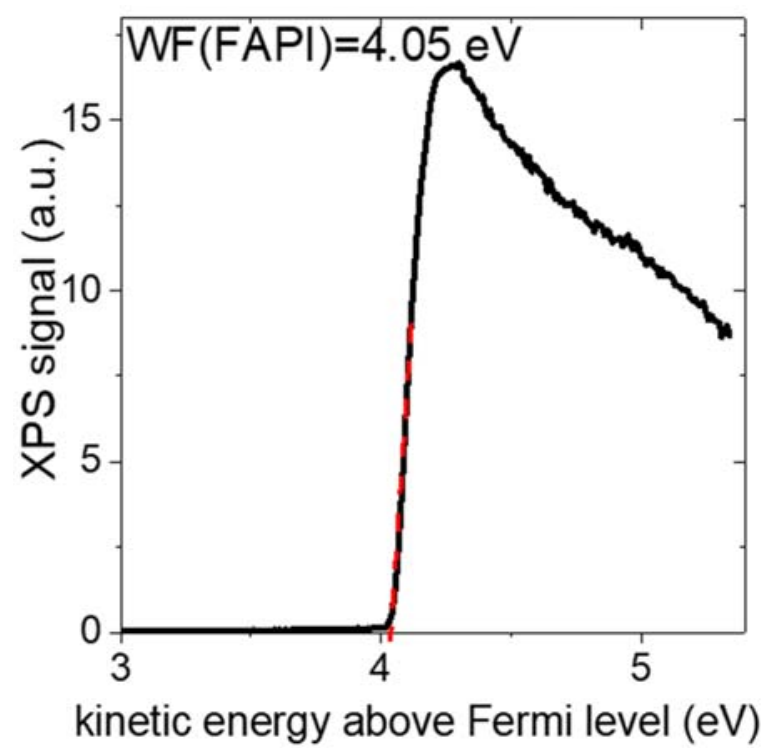

Figure 3 Photoemission signal relative to the cut-off of the secondary electron from a FAPI thin film of nanocrystals recorded using $h v=150 \mathrm{eV}$ photon energy

\subsection{Valence bands}

The low binding energy part of the photoemission spectrum is used to reveal the valance band, see Figure $\mathrm{S} 4$.

Figure $S 4$ Photoemission signal relative to the valence band from a FAPI nanocrystals film taken at $h v=60 \mathrm{eV}(a$.) and $800 \mathrm{eV}(b$.$) .$

The photoemission relative to the valance band of $\mathrm{TiO}_{x}$ and $\mathrm{MoO}_{x}$ is given in Figure $\mathrm{S} 5$. It reveals in particular the presence of oxygen vacancy state within the band gap. ${ }^{1}$


Figure 55 Photoemission signal relative to the valence band of $\operatorname{TiO}_{x}\left(a\right.$.) and $\mathrm{MoO}_{x}($ b.) recorded using hv=150 eV photon energy. 
3.3 Core level overview
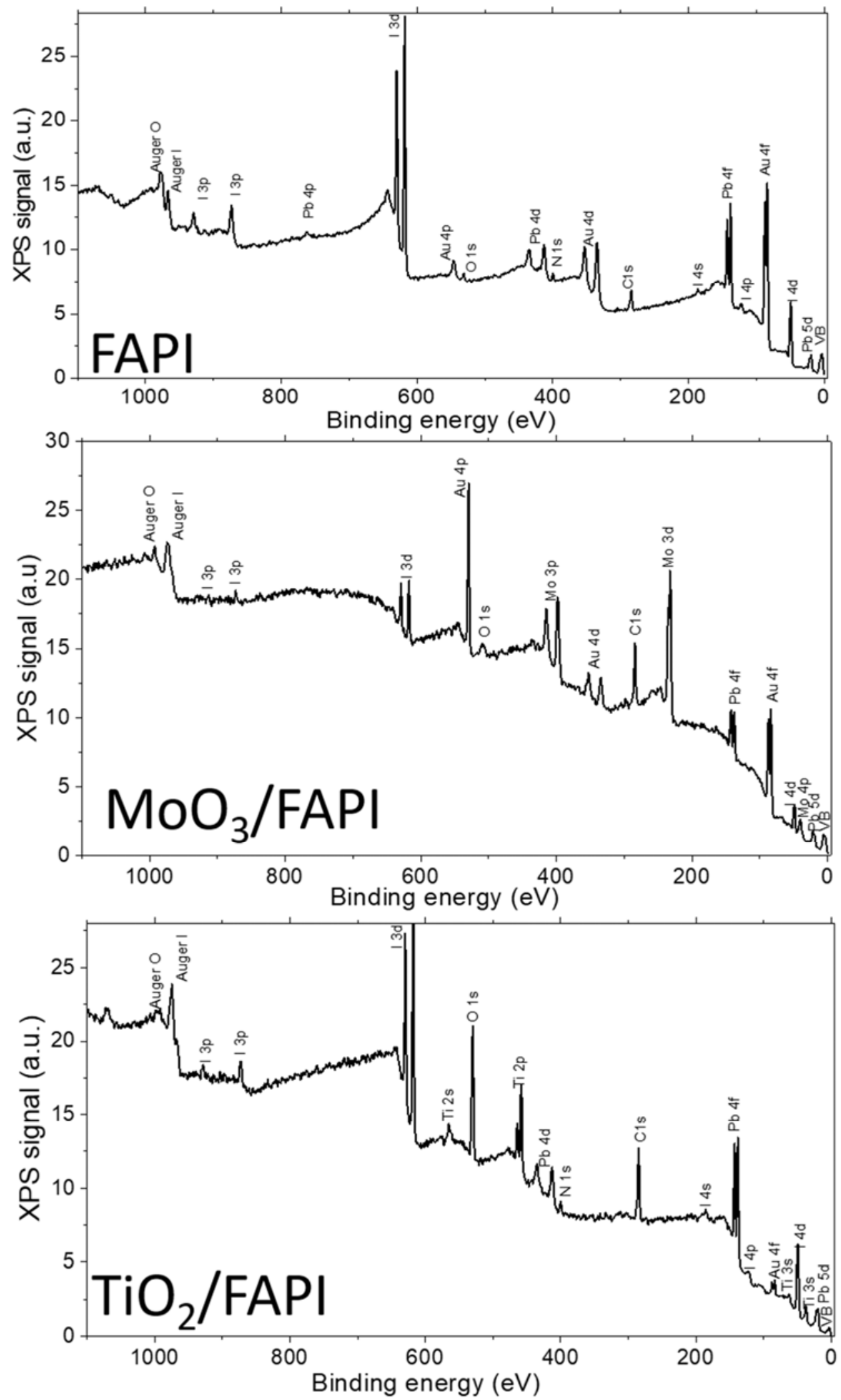

Figure $\mathrm{S} 6$ Photoemission spectrum for a film of FAPI nanocrystals (a), (b) FAPI with $\mathrm{MoO}_{3}$ on the surface and $\mathrm{TiO}_{2}$ on the surface (c) recorded using hv= $1486.6 \mathrm{eV}$ photon energy (Al source). Peak attribution is made using ref ${ }^{2}$. 


\subsection{Analysis of core level for FAPI with and without $\mathrm{TiO}_{x}$ functionalization}

The analysis of the core levels has been done using CasaXPS software. A Shirley-type background has been taken into account for all core levels and the fitting of the different components is done using Gaussian-Lorentzian convolutions.

With $\mathrm{TiO}_{\mathrm{x}}$ functionalization on $\mathrm{FAPI}$, the residual $\mathrm{Pb}^{\circ}$ peak has a vanishing contribution. We also note that the peak relative to $\mathrm{Pb}^{2+}$ is strongly redshifted towards higher binding energy. The binding energy of the $\mathrm{Pb} 4 \mathrm{f}$ state is now $139.12 \mathrm{eV}$ (+0.68 eV compared to pristine FAPI), see Figure S 7b.

Regarding the $\mathrm{TiO}_{x}$, we observe that the pristine material presents two contributions with a binding energy of 457.85 and $459.37 \mathrm{eV}\left(\mathrm{SO}=5.7 \mathrm{eV}\right.$ ), see Figure S 7c. These two contributions can be attributed to $\mathrm{Ti}^{+1 I I}$ and $\mathrm{Ti}^{+\mathrm{IV}}$ respectively. The actual stoichiometry of the compound is found to be $\mathrm{TiO}_{1.8}$. Note that the presence of $\mathrm{Ti}^{+111}$ is resulting from the lack of high temperature $\left(>400^{\circ} \mathrm{C}\right)$ annealing after the $\mathrm{TiO}_{2}$ deposition. Indeed the latter will have irreversibly damaged the FAPI film. After its deposition of FAPI, the $\mathrm{Ti}^{+11 I}$ almost disappears while the peak relative to $\mathrm{Ti}^{+\mathrm{VI}}$ see this binding energy increase by $+0.26 \mathrm{eV}$, see Figure $S 7 d$. This is a clear signature of the material oxidation ${ }^{3}$, or in other word there is an electron transfer from the $\mathrm{TiO}_{2}$ to the $\mathrm{FAPI}$.
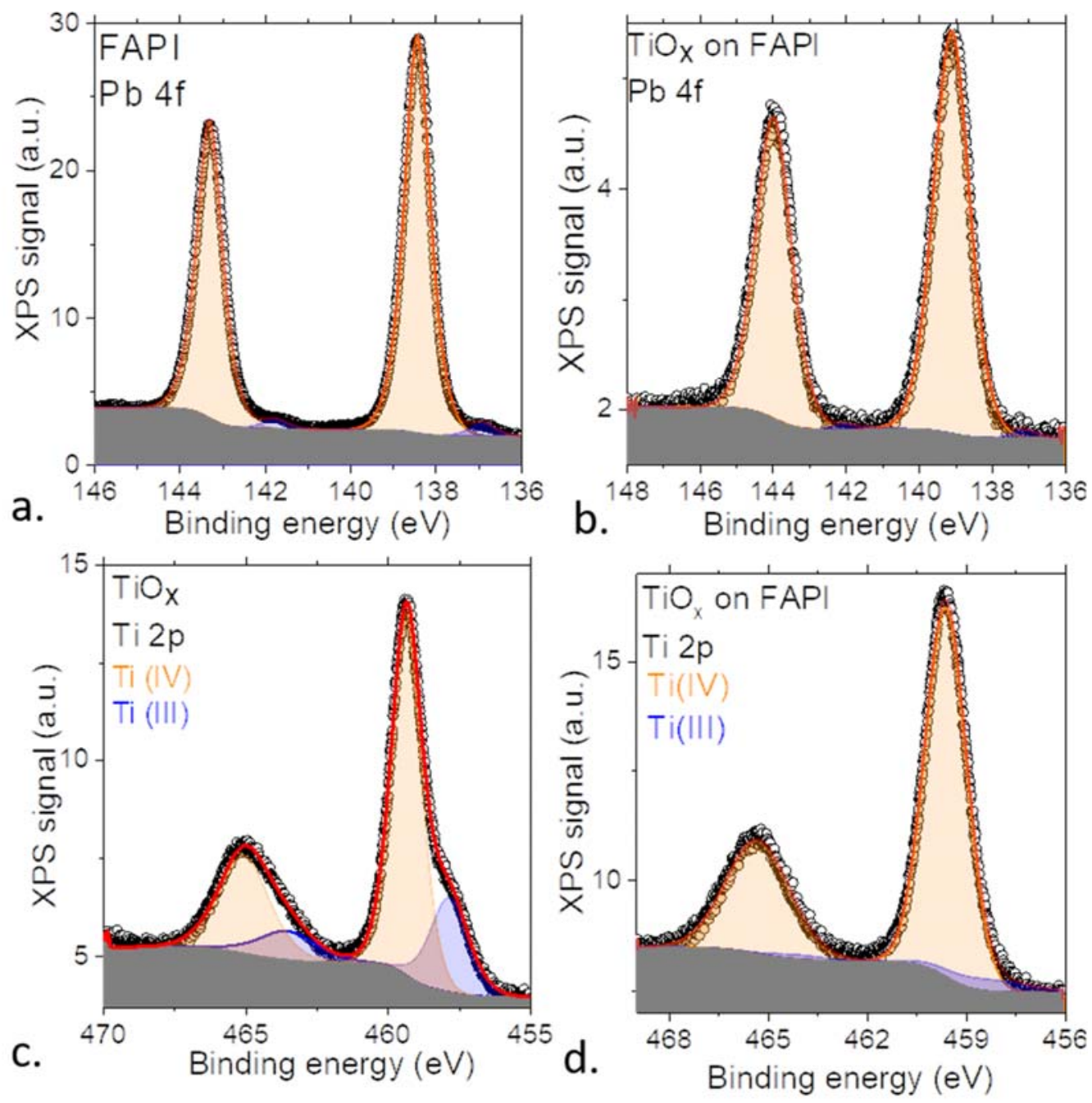

Figure $\mathrm{S} 7$ Photoemission spectrum relative to the $\mathrm{Pb} 4 \mathrm{f}$ state for a film of FAPI nanocrystals with (b) and without (a.) $\mathrm{TiO}_{x}$ deposited on the top. Photoemission spectra relative to the $\mathrm{Ti} 2 \mathrm{p}$ state for a film of $\mathrm{TiO}_{2}$ on gold (c.) and for $\mathrm{TiO}_{x}$ deposited on a FAPI nanocrystals film (d.) 


\subsection{Analisys of core level for FAPI with and without MoOx functionalization}

In pristine $\mathrm{FAPI}$, we can observe two contributions of lead which can be attributed to $\mathrm{Pb}^{2+}$ and $\mathrm{Pb}^{\circ}$, see Figure $\mathrm{S} 8 \mathrm{a}$. The respective binding energies are 136.91 and $138.44 \mathrm{eV}$. In this case the full width at half maximum (FWHM) is found to be $0.73 \mathrm{eV}$ while the spin-orbit coupling energy is taken equal to $4.86 \mathrm{eV}$. After MoOx deposition, the $\mathrm{Pb}^{0}$ disappears, while a second contribution at higher binding energy appears. In this case, the binding energies are found to be 138.26 and $138.9 \mathrm{eV}$ for the two contributions, see Figure $\mathrm{S} 8 \mathrm{~b}$.

Regarding the MoOx, we clearly observe three contributions in the spectrum relative to the Mo $3 \mathrm{~d}$ state. They appear at 230.05, 231.45 and $232.34 \mathrm{eV}$ for peak relative to $\mathrm{Mo}^{\mathrm{IV}}, \mathrm{Mo}^{\mathrm{V}}$ and $\mathrm{Mo}^{\mathrm{VI}}$, see Figure S 8c. The actual stoichiometry of the compound is found to be $\mathrm{MoO}_{2.6}$. Note that the main contribution is associated with the higher oxidation state. The spin orbit is set at $3.2 \mathrm{eV}$ in this case. After its deposition on the FAPI film, we observe a clear change of the relative weight of the different oxidation states. The weight of the $+\mathrm{Vl}$ state is reduced while the weight of the $+\mathrm{V}$ state is increased which is a clear signature of the material reduction, see Figure $\mathrm{S} 8 \mathrm{~d}$. This reduction can be interpreted as an electron transfer from the FAPI to the MoOx layer.
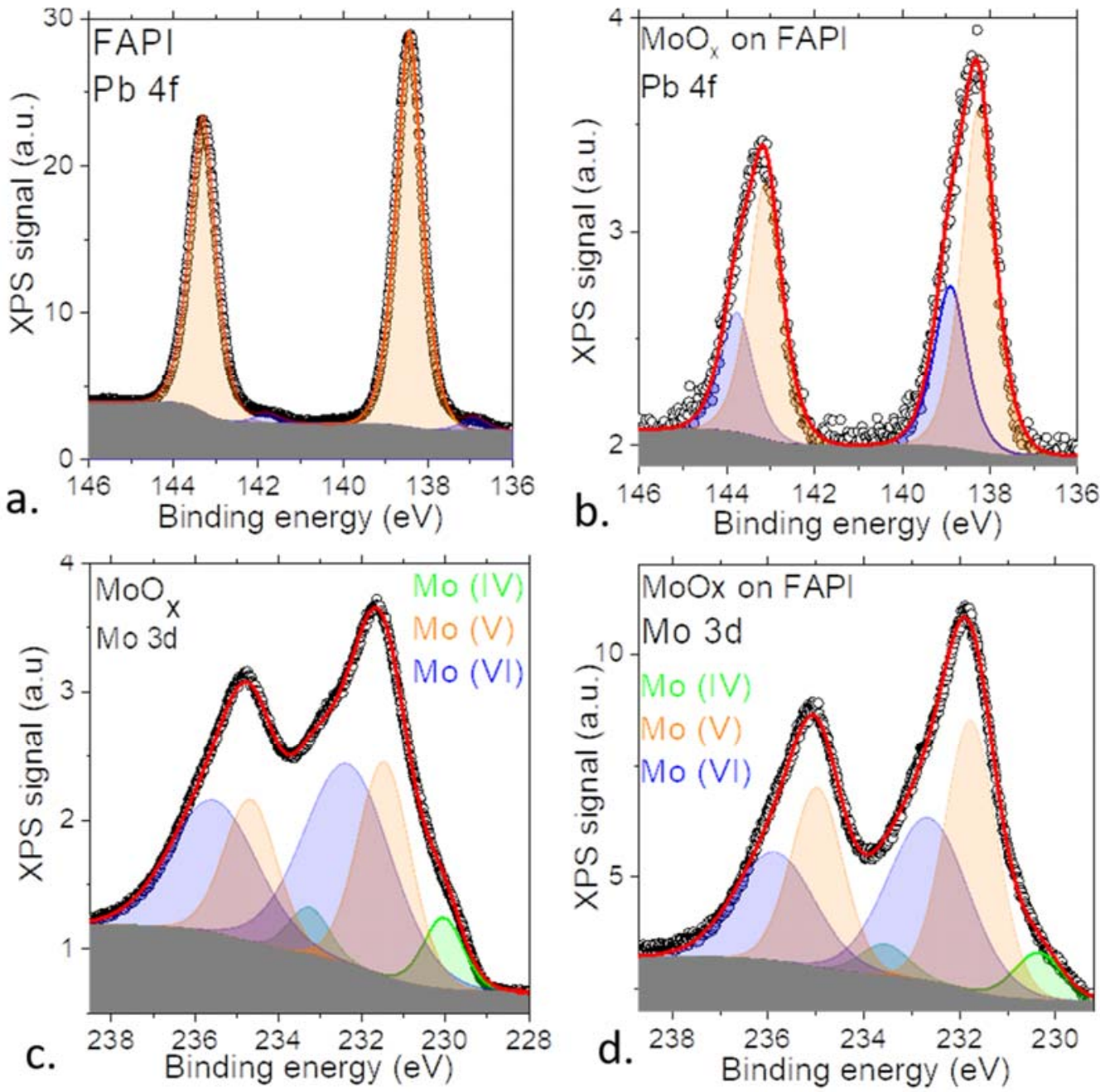

Figure $S 8$ Photoemission spectrum relative to the $\mathrm{Pb} 4 \mathrm{f}$ state for a film of FAPI nanocrystal with (b) and without (a.) MoOx deposited on the top. Photoemission spectra relative to the Mo 3d state for a film of MoOx on gold (c.) and for MoOx deposited on a FAPI nanocrystal film (d.) 


\section{References}

(1) Greiner, M. T.; Chai, L.; Helander, M. G.; Tang, W.-M.; Lu, Z.-H. Transition Metal Oxide Work Functions: The Influence of Cation Oxidation State and Oxygen Vacancies. Advanced Functional Materials 2012, 22, 4557-4568.

(2) Moulder, J. F.; Stickle, W. F.; Sobol, P. E.; Bomben, K. D. Handbook of X-Ray Photoelectron Spectroscopy, PerkinElmer Corp.; Eden Prairie: MN, 1992.

(3) Greiner, M. T.; Lu, Z.-H. Thin-Film Metal Oxides in Organic Semiconductor Devices: Their Electronic Structures, Work Functions and Interfaces. NPG Asia Mater 2013, 5, e55. 